Horst Weishaupt

\title{
Zur Situation sonderpädagogischer Förderung in Hessen im Schuljahr 2016/17
}

\section{Zusammenfassung}

Die Schulstatistik von Hessen weist alle Schüler*innen nach ihrer besuchten Schule - sei es eine allgemeine oder eine Förderschule - und ihrem Wohnort sowie bei einer sonderpädagogischen Förderung auch nach ihrem Förderschwerpunkt aus. Mit diesen Daten wird die aktuelle Fördersituation an Förderschulen analysiert. Zudem werden einige schulorganisatorische Bedingungen der Inklusion von Schüler ${ }^{*}$ innen mit sonderpädagogischem Förderbedarf an allgemeinen Schulen untersucht und ergänzend das Verhältnis von an Förderschulen und integrativ unterrichteten Schüler*innen. Abschließend wird die Gesamtsituation sonderpädagogischer Förderung in Hessen kurz gewürdigt.

Schlüsselwörter: Inklusion, Förderschule, sonderpädagogische Förderung, Sonderpädagogische Förderschwerpunkte, regionale Disparitäten, kumulative Herausforderungen an Schulen

\section{The Situation of Special Needs Education in Hessen in the School Year 2016/17}

\begin{abstract}
Hessian school statistics identify all students with regard to their attended school general schooling or special needs education -, the place they live, and their specific educational needs types, as the case may be. Based on these data, this article analyzes the current situation at special schools. Furthermore, it examines some school-organizational conditions referring to the inclusion of students with special educational needs in general schools as well as the proportion of students attending both special schools and inclusive schooling. Finally, it shortly comments on the overall situation of special needs education in Hessen

Keywords: inclusion, special schools, special needs education, special educational needs types, regional disparities, cumulative challenges at schools
\end{abstract}




\section{Einleitung}

Mit der Unterzeichnung der UN-Behindertenrechtskonvention im Jahr 2008 begann in Deutschland eine neue Phase der Entscheidung über den Förderort von Schüler^innen mit einem sonderpädagogischen Förderbedarf. Erwartet wurde die Schließung einer zunehmenden Zahl von Förderschulen, weil die Unterrichtung und Betreuung von sonderpädagogisch förderungsbedürftigen Kindern im Regelschulsystem als vorherrschende Form der sonderpädagogischen Förderung angestrebt werden sollte. Wie die bundesweite Entwicklung zeigt, ist Deutschland hiervon noch weit entfernt. Zwar ist seit 2009 die Inklusionsquote verstärkt angestiegen und erhöhte sich seit 2000 um 2 Prozent; die Förderschulbesuchsquote ist jedoch nicht im gleichen Umfang gesunken. Sie bewegte sich in den letzten 15 Jahren zwischen 4,5 und 4,9 Prozent. Insgesamt ist damit die Quote der sonderpädagogisch Geförderten im gleichen Zeitraum um 2 Prozent angestiegen und liegt inzwischen bei über 7 Prozent. Zwischen den Ländern der Bundesrepublik gibt es große Abweichungen von dieser durchschnittlichen Entwicklung (Autorengruppe Bildungsberichterstattung, 2016, S. 81).

Hessen soll nachfolgend näher analysiert werden, um mit den dort verfügbaren differenzierten Daten der Schulstatistik die bisherige Entwicklung der sonderpädagogischen Förderung im Schulstufen- und Regionalvergleich zu erfassen. Hessen zeichnet sich insgesamt durch eine unterdurchschnittliche sonderpädagogische Förderquote und eine hinhaltende Umsetzung der UN-Behindertenrechtskonvention aus. Die Förderschulen haben weiterhin eine gesicherte Schülerschaft - was sich in fast konstanten Schülerzahlen ausdrückt -, und die Förderschulbesuchsquoten sind seit 2009 nur leicht rückläufig und immer noch höher als im Jahr 2000. Das Land hat regionale und überregionale Beratungs- und Förderzentren, die den allgemeinen Schulen mit Inklusionsschüler*innen beratend zur Verfügung stehen.

Durch die niedrige Inklusionsquote liegt der Inklusionsanteil in Hessen mit einem Viertel deutlich unter dem Bundesdurchschnitt von fast 38 Prozent (vgl. Tab. 1). In Hessen ist der Inklusionsanteil im Förderschwerpunkt „Emotionale und soziale Entwicklung“ vergleichsweise hoch. Eine wenige Jahre zurückliegende Analyse der Förderschulsituation (Weishaupt, 2017a) ergab auch beim Förderschulangebot einige Besonderheiten. Vor allem war auffällig, dass viele Förderschulen Schüler*innen mit unterschiedlichen Förderschwerpunkten unterrichten und nicht selten zielgleich und zieldifferent zu unterrichtende Förderschüler*innen gemeinsam unterrichtet wurden. 
Tab. 1: Sonderpädagogische Förderquote, Förderschulbesuchsquote und Inklusionsanteil in Deutschland insgesamt und in Hessen im Schuljahr 2015/16 nach sonderpädagogischen Förderschwerpunkten

\begin{tabular}{|c|c|c|c|c|c|c|}
\hline \multirow[b]{2}{*}{ Förderschwerpunkte } & \multicolumn{3}{|c|}{ Deutschland } & \multicolumn{3}{|c|}{ Hessen } \\
\hline & 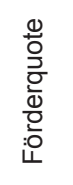 & 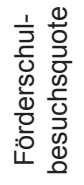 & 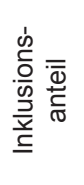 & 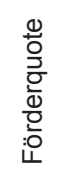 & 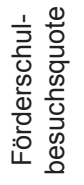 & 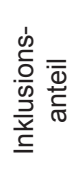 \\
\hline Insgesamt & 7,11 & 4,43 & 37,7 & 5,70 & 4,25 & 25,4 \\
\hline Förderschwerpunkt Lernen & 2,61 & 1,44 & 45,0 & 2,39 & 1,66 & 30,6 \\
\hline $\begin{array}{l}\text { sonstige Förderschwerpunkte } \\
\text { zusammen }\end{array}$ & 4,49 & 2,99 & 33,4 & 3,31 & 2,59 & 21,6 \\
\hline Sehen & 0,11 & 0,06 & 42,5 & 0,07 & 0,06 & 19,7 \\
\hline Hören & 0,26 & 0,14 & 45,1 & 0,16 & 0,13 & 17,0 \\
\hline Sprache & 0,77 & 0,42 & 45,5 & 0,60 & 0,47 & 21,3 \\
\hline $\begin{array}{l}\text { Körperliche und motorische } \\
\text { Entwicklung }\end{array}$ & 0,50 & 0,33 & 34,1 & 0,33 & 0,24 & 28,4 \\
\hline Geistige Entwicklung & 1,16 & 1,03 & 10,7 & 1,02 & 0,92 & 10,2 \\
\hline $\begin{array}{l}\text { Emotionale und soziale } \\
\text { Entwicklung }\end{array}$ & 1,18 & 0,52 & 55,6 & 0,71 & 0,38 & 47,0 \\
\hline Kranke & 0,16 & 0,14 & 8,4 & 0,39 & 0,37 & 3,5 \\
\hline $\begin{array}{l}\text { Förderschwerpunkt } \\
\text { übergreifend }\end{array}$ & 0,04 & 0,03 & 16,9 & & & \\
\hline $\begin{array}{l}\text { Lernen, Sprache, emotionale } \\
\text { und soziale Entwicklung (LSE) }\end{array}$ & 0,16 & 0,16 & 0,0 & & & \\
\hline $\begin{array}{l}\text { noch keinem Förder- } \\
\text { schwerpunkt zugeordnet }\end{array}$ & 0,15 & 0,13 & 9,2 & 0,03 & 0,03 & 0,0 \\
\hline
\end{tabular}

Quelle: KMK, 2016a, 2016b; eigene Berechnungen

An diese Ergebnisse anknüpfend wird nachfolgend die aktuelle Gesamtsituation sonderpädagogischer Förderung in Hessen analysiert. Damit soll bewusst eine in der gegenwärtigen Forschung vorherrschende Konzentration auf das Inklusionsthema (s. z. B. Moser \& Lütje-Klose, 2016) vermieden und eine über die gängigen Indikatoren (Brüggemann \& Tegge, 2016) hinausgehende differenzierte schulstatistische Beschreibung der sonderpädagogischen Förderung angestrebt werden. Dabei sind mehr die schulorganisatorischen Rahmenbedingungen und die Betreuungssituation der Schüler ${ }^{\star}$ innen mit festgestelltem sonderpädagogischem Förderbedarf Gegenstand der Analysen als der Unterricht und beispielsweise die Probleme der Lehrerkooperation, die relativ stark die gegenwärtige Diskussion bestimmen. ${ }^{1}$ Ermöglicht

1 Zur Lehrkräftesituation an den Schulen wurden keine Daten herangezogen, da hierzu weitere Sonderauswertungen der Schulstatistik notwendig gewesen wären. 
werden die Analysen dadurch, dass in der hessischen Schulstatistik neben der besuchten Schule auch der Wohnort der Schüler*innen erfasst wird. So können die Auswirkungen der regional ungleichen Verteilung von Förderschulen der verschiedenen Förderschwerpunkte auf die Diagnose sonderpädagogischen Förderbedarfs betrachtet werden. Erwartet wird in Kreisen ohne eine Förderschule mit einem entsprechenden Förderschwerpunkt ein niedrigerer Anteil von Schüler*innen mit einem entsprechenden sonderpädagogischen Förderbedarf. Unter dieser Voraussetzung könnte Inklusion eine schulorganisatorische Maßnahme sein, um Lücken im Förderschulangebot zu kompensieren und eine flächendeckend einheitliche sonderpädagogische Versorgung in allen Förderschwerpunkten zu erreichen.

Ob Inklusion tatsächlich zu einem Ausgleich der sonderpädagogischen Förderquoten beiträgt, ist eine weitere Untersuchungsfrage. Schließlich interessiert, ob sich Inklusionsschulen auch hinsichtlich anderer Merkmale der Schülerzusammensetzung von sonstigen Schulen unterscheiden. Dazu wird zunächst die Fördersituation für die Schüler ${ }^{\star}$ innen an Förderschulen beschrieben (1.), um dann zu analysieren, wie sich dazu die Situation inklusiver Unterrichtung für die Schüler*innen verhält (2.). Abschließend wird die Gesamtsituation sonderpädagogischer Förderung unter dem Gesichtspunkt der Fördersituation für die Schüler*innen analysiert (3.), um die bisherige Entwicklung nach Verabschiedung der UN-Behindertenrechtskonvention danach zu bewerten, welche Veränderungen sie bei der Beschulung von Schüler*innen mit sonderpädagogischem Förderbedarf ausgelöst hat (4.).

\section{Sonderpädagogische Förderung an den Förderschulen in Hessen}

Einleitend wurde bereits darauf verwiesen, dass die bisherige Entwicklung kaum das vor zehn Jahren bereits bestehende Förderschulangebot berührte. In Hessen ging zwischen 2006 und 2016 die Zahl der Förderschulen nur um 10 Schulen auf 242 zurück. Die Schülerzahl an Förderschulen verringerte sich zwar um 17 Prozent, doch nahm auch die Schülerzahl im Pflichtschulalter insgesamt um 10 Prozent ab. Charakteristisch für die Förderschulsituation in den Landkreisen Hessens (im Gegensatz zu den Großstädten) ist die Verbindung von nahezu jeder fünften Förderschule (von insgesamt 193) mit allgemeinen Schulen: 20 mit Grundschulen, 13 mit Gesamtschulen (darunter eine mit Grundschulzweig) und 1 Mittelstufenschule. Schließlich gibt es eine private städtische Förderschule mit Gymnasialzweig. Hessen hat - wie auch andere Länder - kein schulorganisatorisch grundsätzlich vom Regelschulsystem isoliertes Förderschulangebot.

Mit einer durchschnittlichen Schülerzahl von 89 (vgl. Tab. 2) können in Hessen die meisten Förderschulen nur eine Lerngruppe je Jahrgang bilden. Dies hat zugleich Auswirkungen auf die Zahl der Lehrkräfte an den einzelnen Schulen, die - differen- 


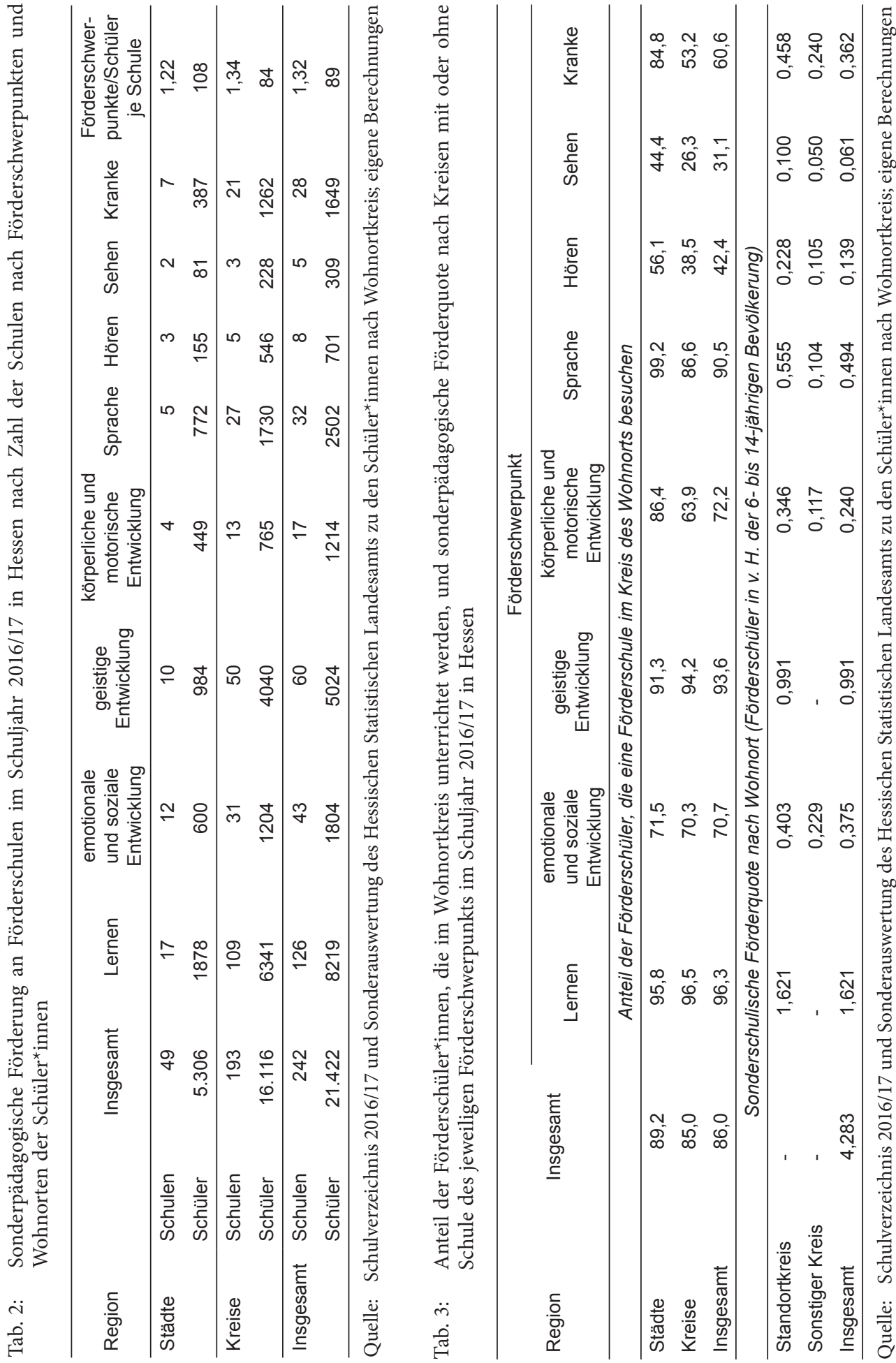


zierte Analysen dazu fehlen, wären aber sehr wünschenswert - kaum die notwendige Breite an fachlicher Expertise an den Schulen aufweisen können, um in allen Fächern einen Unterricht zu erteilen, der zielgleich zu unterrichtende Schüler ${ }^{\star}$ innen über einen Hauptschulabschluss hinausführen könnte. ${ }^{2}$ Hinzu kommt, dass an vielen Schulen Schüler*innen nicht nur eines Förderschwerpunkts unterrichtet werden, sondern auch Schüler*innen, die in anderen Schwerpunkten sonderpädagogisch gefördert werden. Nicht selten werden Schüler*innen sowohl zielgleich als auch zieldifferent gemeinsam unterrichtet. Immerhin 30 Prozent der Förderschüler*innen in Hessen wurden im Jahr 2014 in Förderschulen mit zwei und mehr Förderschwerpunkten unterrichtet (Weishaupt, 2017a, S. 48). Die 49 Förderschulen in Städten hatten Schüler*innen in 60 Förderschwerpunkten; sie unterrichteten durchschnittlich Schülerinnen in 1,22 Förderschwerpunkten. Die 193 Förderschulen in den Kreisen unterrichteten sogar Schüler*innen in 259 Förderschwerpunkten und damit durchschnittlich in 1,32 Förderschwerpunkten (vgl. Tab. 2). Dabei sind die Schüler*innen mit mehreren Förderschwerpunkten nicht berücksichtigt, die von der hessischen Schulstatistik nur nach dem Haupt-Förderschwerpunkt erfasst werden. $\mathrm{Zu}$ berücksichtigen ist zusätzlich, dass es auch bei einem prinzipiell zielgleich zu unterrichtenden Förderschwerpunkt Schülerinnen gibt, die zieldifferent $\mathrm{zu}$ unterrichten sind. 2011 waren dies in Nordrhein-Westfalen 8 Prozent der Schüler*innen in zielgleich zu unterrichtenden Förderschwerpunkten (s. Weishaupt, 2017a, S. 42 f.). Es ist jedenfalls davon auszugehen, dass in vielen Förderschulklassen ein binnendifferenzierter Unterricht erteilt werden muss, um den unterschiedlichen sonderpädagogischen Förderbedarfen der Schüler*innen gerecht werden zu können. Dies wirft die Frage auf, weshalb trotz dieser heterogenen Lernbedingungen an vielen Förderschulen an ihnen festgehalten wird. Es ist eines der großen Versäumnisse der Schulforschung der letzten Jahrzehnte, den Lehr-/Lernbedingungen an Förderschulen kaum Aufmerksamkeit geschenkt zu haben (ebd.).

Trotz der Bemühungen, kleine Förderschulen zu erhalten, kann eine über alle Kreise hinweg einheitliche flächendeckende Versorgung mit dem bestehenden Förderschulangebot nicht erreicht werden. Nur in den Förderschwerpunkten „Lernen“ und "Geistige Entwicklung“ gibt es in Hessen Förderschulen in allen Kreisen. In den Schwerpunkten „Hören“, „Körperlich-motorische Entwicklung“ und „Sehen“ existieren sogar deutlich weniger Förderschulen als Kreise und kreisfreie Städte (26). Da die Schulstatistik sowohl den Schulort als auch den Wohnort der Schüler*innen der Förderschulen erfasst, kann die wohnortbezogene Versorgungssituation nach Kreisen und Förderschwerpunkten analysiert werden (vgl. Tab. 3). Im Ergebnis zeigt sich, dass in den beiden Förderschwerpunkten mit Schulen in allen Kreisen mehr als 90 Prozent der Förderschüler*innen innerhalb des Wohnortkreises unterrichtet werden. In den

2 Von den 768 Absolvent*innen von Förderschulen 2015/16 ohne einen schulartspezifischen Abschluss erreichten nur 21,4 Prozent einen Realschulabschluss und nur 1 Prozent eine Hochschulzugangsberechtigung (Hessisches Statistisches Landesamt, 2017; eigene Berechnung). 
anderen Förderschwerpunkten ist dieser Anteil deutlich niedriger und meist in den Städten höher als in den Landkreisen mit einem eingeschränkten Förderschulangebot. Eine Ausnahme bildet der sonderpädagogische Förderschwerpunkt „Sprache“, in dem der günstig erscheinende hohe Anteil von Schüler*innen, die innerhalb des Kreises wohnen, durch eine extrem niedrige Förderquote außerhalb der Standortkreise erreicht wird: In den Standortkreisen von Förderschulen mit dem Schwerpunkt "Sprache" ist die Förderquote fünfmal so hoch wie in den Kreisen ohne eine Förderschule dieses Schwerpunkts. Auch in den anderen Förderschwerpunkten sinkt die Förderquote um mindestens etwa die Hälfte, wenn in dem Wohnortkreis keine Förderschule des entsprechenden Förderschwerpunkts vorhanden ist. Die Diagnose des sonderpädagogischen Förderbedarfs scheint sich somit weniger an dem Förderbedarf der Schüler*innen als an der Verfügbarkeit eines entsprechenden Förderschulangebots auszurichten. Andernfalls sind die großen regionalen wohnortbezogenen Unterschiede kaum erklärbar.

Da die Förderschule in Hessen nur in den Förderschwerpunkten „Lernen“ und "Geistige Entwicklung" in der Lage ist, eine flächendeckende Versorgung auf Kreisebene sicherzustellen, könnten die Versorgungsunterschiede in den anderen Förderschwerpunkten durch eine inklusive Beschulung verringert werden. Daher wird anschließend überprüft, ob durch eine inklusive Unterrichtung von Schüler*innen in den Kreisen ohne Förderschulangebot diese Disparitäten tatsächlich geringer werden.

\section{Schülerinnen mit sonderpädagogischem Förderbedarf an allgemeinen Schulen}

Schüler*innen an allgemeinen Schulen werden in den Schularten des gegliederten Schulsystems unterrichtet. Nachfolgend wird zwischen Grundschüler*innen und Schüler*innen in den Schularten der Sekundarstufe I unterschieden. Die 44 Schüle$r^{\star}$ innen in der Sekundarstufe II an allgemeinen Schulen in Hessen bleiben unberücksichtigt. Ebenfalls wird wieder zwischen den fünf Großstädten und den 21 Landkreisen differenziert. Grundlage der Analyse bilden neben Daten der Schulstatistik (Hessisches Statistisches Landesamt, 2017) Daten des Schulverzeichnisses, das Angaben zu den Schüler*innen nach ihrem Förderschwerpunkt und zu der besuchten Schulart an den einzelnen Schulen enthält.

In Hessen entspricht die Zahl der inklusiv unterrichtenden Schulen mit 813 Grundschulen und 384 weiterführende Schulen in etwa zwei Dritteln aller allgemeinen Schulen (vgl. Tab. 4) und damit dem Bundesdurchschnitt (Autorengruppe Bildungsberichterstattung, 2018, S. 106). Der Anteil der Schulen mit Inklusionsschülerinnen ist im Grundschulbereich in den Großstädten mit drei Vierteln aller Schulen deutlich höher als in den Landkreisen (zwei Drittel der Schulen). In der Sekundarstufe I haben 


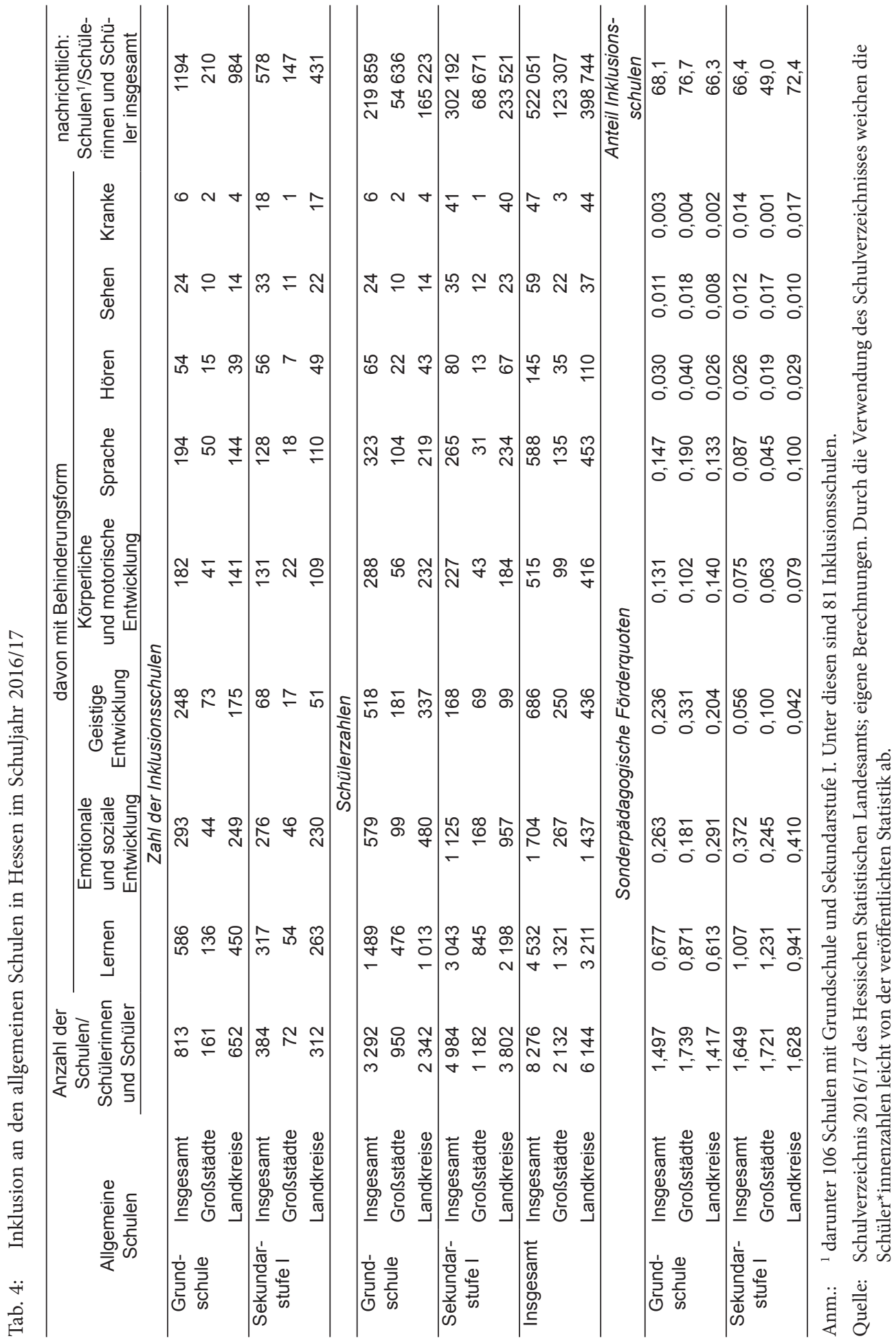


in den Landkreisen über 70 Prozent der Schulen Inklusionsschüler*innen, während in den Großstädten nur weniger als die Hälfte der Schulen Inklusionsschüler*innen aufgenommen hat.

Auffällig ist, dass an Schulen aller weiterführenden Schularten Inklusionsschüler*innen in allen sonderpädagogischen Förderschwerpunkten unterrichtet werden. Der Anteil der Inklusionsschüler*innen an der Gesamtzahl der Schüler*innen variiert dabei zwischen 0,1 Prozent an Gymnasien, 0,5 Prozent an Realschulen, 2,8 Prozent an Integrierten Gesamtschulen und 7,5 Prozent an Hauptschulen. Der Anteil der zieldifferent zu unterrichtenden Schüler*innen in den Förderschwerpunkten "Lernen“ und „Geistige Entwicklung“ an allen Inklusionsschüler*innen schwankt zwischen 8,9 Prozent am Gymnasium, 16,7 Prozent an den Realschulen, 68,8 Prozent an Integrierten Gesamtschulen und 76,4 Prozent an Hauptschulen (Angaben ohne Tabelle). An den Grundschulen liegt der Anteil der Inklusionsschüler*innen landesweit bei 1,5 Prozent, davon 61 Prozent in den zieldifferent zu unterrichtenden Förderschwerpunkten (vgl. Tab. 4).

Betrachtet man die Inklusionsquoten nach Schulstufen und Regionen, zeigt sich in der Sekundarstufe I eine höhere Inklusionsquote als in der Grundschule und in den Großstädten eine höhere als in den Landkreisen. Beide Ergebnisse sind unerwartet. Die ungünstigere Erreichbarkeit von Förderschulen im ländlichen Raum sollte dort eigentlich die Inklusion begünstigen. In der Inklusionsdiskussion wird ebenfalls davon ausgegangen, dass zunehmend Schulanfänger*innen sofort inklusiv unterrichtet werden und von den Grundschulkassen ausgehend der Inklusionsprozess voranschreitet. Möglicherweise verhindern die schon angesprochenen personellen Restriktionen und die Bestandserhaltungsinteressen von Förderschulen eine Erhöhung der Inklusionsquoten bereits in den Grundschulkassen. ${ }^{3}$

Deutlich abweichend von dem allgemeinen Befund ist im Förderschwerpunkt „Emotionale und soziale Entwicklung“ die Inklusionsquote in den Landkreisen höher als in den Großstädten. Nur im Förderschwerpunkt „Geistige Entwicklung“ konzentrieren sich die Inklusionsschüler*innen im Grundschulbereich.

Angesichts der vielen Inklusionsschulen, aber einer insgesamt niedrigen Inklusionsquote, stellt sich die Frage nach der Zahl der Inklusionsschüler*innen je Schule. Im Grundschulbereich besuchen durchschnittlich 4,1 Schüler*innen mit einem sonderpädagogischen Förderbedarf eine Inklusionsschule (5,9 in den Großstädten und 3,6 in den Landkreisen). In der Sekundarstufe I sind es 12,9 Schüler ${ }^{\star}$ innen (16,4 in den Großstädten und 12,1 in den Landkreisen). An einem Viertel der Grundschulen mit Inklusionsschüler*innen (211) und an 5,7 Prozent (22) der Sekundar-

3 In Hessen wurden in den letzten zehn Jahren konstant 2,5 Prozent aller Schulanfänger*innen direkt in Förderschulen eingeschult (Autorengruppe Bildungsberichterstattung, 2018, Tab. C5-9web). 
stufenschulen wird nur eine Schülerin bzw. ein Schüler mit einem sonderpädagogischen Förderbedarf unterrichtet. Diese Form der Einzelintegration mag für die Außendarstellung der Schulen wichtig sein, dürfte aber eine angemessene sonderpädagogische Betreuung der Schüler*innen erheblich erschweren, weil es kaum möglich sein dürfte, mit vertretbarem Aufwand sonderpädagogisches Personal an diesen Schulen zur Verfügung zu stellen.

In der öffentlichen Diskussion wird oft auf die starke Belastung der Lehrer ${ }^{\star}$ innen durch mehrere Schülerinnen mit einem sonderpädagogischen Förderbedarf in einer Klasse hingewiesen. ${ }^{4}$ Das dürfte an den hessischen Schulen aber die Ausnahme sein, denn an den meisten Schulen lässt die Gesamtzahl dieser Schüler*innen nicht auf mehrere von ihnen in einem Jahrgang schließen. Auch ist nicht bekannt, ob die Schulen bei mehreren Schüler*innen mit einem sonderpädagogischen Förderbedarf diese in einer Klasse konzentrieren oder über mehrere Parallelklassen verteilen. Nur 104 der insgesamt 1.116 Inklusionsschulen in Hessen unterrichten mehr als 19 Schüler*innen mit einem sonderpädagogischen Förderbedarf.

Vor allem im Grundschulbereich unterrichten zahlreiche Schulen ausschließlich Inklusionsschüler*innen eines sonderpädagogischen Förderschwerpunktes. Dies trifft auf 29 Prozent der großstädtischen und 45 Prozent der ländlichen Inklusionsgrundschulen zu. Dadurch kommen die allgemeinen Schulen nur sehr begrenzt als Alternative zu den Förderschulen in Frage. Dies mag aber auch mit Problemen einer angemessenen Personalausstattung mit sonderpädagogischen Lehrkräften zusammenhängen. Bei einem angenommenen durchschnittlichen zusätzlichen Stundenbedarf je Schüler*in mit einem sonderpädagogischen Förderbedarf von fünf Unterrichtsstunden benötigt eine Schule wenigstens fünf Inklusionsschüler*innen für eine zusätzliche sonderpädagogische Vollzeitkraft. Nur drei von zehn Inklusionsgrundschulen erfüllen diese Bedingung. In der Sekundarstufe I ist es nur ein Viertel der Schulen, die weniger als fünf Inklusionsschüler*innen unterrichten.

Eine Ursache dafür, dass gerade an den eher kleineren Grundschulen in den Landkreisen weniger häufig Schüler*innen inklusiv unterrichtet werden, obwohl dort die Schulwege zur nächsten Förderschule vermutlich durchschnittlich deutlich weiter sind als in einer Großstadt, dürfte an der fehlenden Verfügbarkeit von Sonderpädagog*innen liegen. Es wäre sehr informativ zur Beurteilung der Situation sonderpädagogischer Förderung, wenn den konkreten unterrichtlichen Bedingungen für die einzelnen Schüler*innen an den allgemeinen Schulen nachgegangen werden könnte (Verteilung der Schülerinnen mit einem sonderpädagogischen Förderbedarf auf die einzelnen Klassen, Bereitstellung und Nutzung von sonderpädagogischem Personal, Fragen der Unterrichtsorganisation etc.).

4 Nicht ausgeschlossen ist eine hohe Belastung durch Kinder, die einen nicht anerkannten sonderpädagogischen Förderbedarf haben. 
Für eine erfolgreiche Inklusion von Schüler*innen können weitere Organisationsmerkmale von Schulen bedeutsam sein. Da die Förderschulen in Hessen überwiegend gebundene Ganztagsschulen sind, ist ein Ganztagsangebot an allgemeinen Schulen vermutlich für Eltern eine wichtige Vorbedingung, um eine inklusive Förderung ihrer Kinder in Erwägung zu ziehen. Dies bestätigt eine entsprechende Analyse des Schulverzeichnisses (vgl. Tab. 5). Mehr als die Hälfte der Inklusionsgrundschulen haben Ganztagsangebote (nach der Definition des Kultusministeriums), ${ }^{5}$ und bei den Schulen der Sekundarstufe I sind es in den Großstädten über 80 Prozent und in den Landkreisen über 90 Prozent. Die Schulen ohne Schülerinnen mit einem sonderpädagogischen Förderbedarf haben in allen unterschiedenen Gruppen deutlich niedrigere Anteile von Ganztagsschulen.

Tab. 5: Unterschiede in den Organisationsmerkmalen zwischen Schulen mit Inklusionsschüler*innen (Inklusionsschulen) und sonstigen Schulen nach Region und Schulstufe 2016/17

\begin{tabular}{llcccc}
\hline \multirow{2}{*}{$\begin{array}{l}\text { Organisations- } \\
\text { merkmal }\end{array}$} & Region & \multicolumn{2}{c}{ Grundschule } & \multicolumn{2}{c}{ Sekundarstufe I } \\
\cline { 3 - 6 } & & $\begin{array}{c}\text { Inklusions- } \\
\text { schule }\end{array}$ & Sonstige & $\begin{array}{c}\text { Inklusions- } \\
\text { schule }\end{array}$ & Sonstige \\
\hline $\begin{array}{l}\text { Ganztags- } \\
\text { schule }\end{array}$ & Großstädte & 56,2 & 30,6 & 80,6 & 54,6 \\
\hline Flüchtlings- & Landkreise & 55,1 & 34,2 & 91,5 & 58,3 \\
klassen & Großstädte & 27,2 & 8,2 & 62,5 & 25,3 \\
\hline Migrations- & Gandkreise & 30,0 & 12,1 & 68,0 & 22,6 \\
anteil1 & Großstädte & 54,9 & 35,2 & 53,2 & 43,3 \\
\hline
\end{tabular}

Anm.: $\quad{ }^{1}$ Anteil der Schülerinnen und Schüler mit Migrationshintergrund. Nach der Definition der Schulstatistik, die den Anteil verglichen mit der Bevölkerungsstatistik um etwa ein Viertel unterschätzt. $93 \%$ der von der Schulstatistik erfassten Migrant ${ }^{*}$ innen haben eine nicht deutsche Familiensprache.

Quelle: Schulverzeichnis 2016/17 des Hessischen Statistischen Landesamts; eigene Berechnungen

5 Hessen unterscheidet Ganztagschulen mit (1) Ganztagsangeboten nach dem Profil 1, die an mindestens drei Wochentagen bis 14:30 Uhr Hausaufgabenbetreuung, Fördermaßnahmen sowie erweiterte Angebote im Wahl- und Freizeitbereich anbieten, und (2) Schulen mit Ganztagsangeboten nach dem Profil 2, die an allen fünf Schultagen pro Woche freiwillige Zusatzangebote von 7:30 Uhr bis 16:00 oder 17:00 Uhr anbieten; unter anderem werden Förderkurse, Wahlangebote sowie den Unterricht ergänzende und erweiternde Arbeitsgemeinschaften und Projekte, die Betreuung von Hausaufgaben und Stillarbeit sowie die Teilnahme an offenen Sport- und Spielgruppen gewährleistet. (3) Nur Profil 3 gewähreistet als gebundene Ganztagsschule eine ganztägige Betreuung während der Schulzeit. Diese verbindliche Ganztagsbetreuung bestand 2015/16 in Hessen nur für 13,5 Prozent der 7- bis 9-jährigen Schüler*innen (Weishaupt, 2017c). 
Während Ganztagsangebote für Inklusionsprozesse förderlich sein können, bedeuten Kinderarmut, Kinder mit nicht deutscher Familiensprache und Intensivklassen für Flüchtlingskinder an den Schulen zusätzliche Herausforderungen für die Lehrkräfte. Die Lehrkräfte an Schulen mit mehrfachen Herausforderungen sind damit zugleich mit sehr unterschiedlichen pädagogischen Anforderungen konfrontiert, die sich kaum im Rahmen des normalen Unterrichtsprogramms bewältigen lassen. Umso bemerkenswerter ist der Befund, dass Inklusionsschulen deutlich häufiger Intensivklassen ${ }^{6}$ aufweisen als Schulen ohne Inklusionsschüler*innen. In der Sekundarstufe I ist der Unterschied besonders deutlich: Zwei Drittel der Inklusionsschulen haben Intensivklassen, von den sonstigen Schulen weniger als ein Viertel.

Zur Armutssituation an den hessischen Schulen gibt es keine verfügbaren Daten. Doch ist der Zusammenhang zwischen Kinderarmut und Migrationsstatus vielfach belegt (Autorengruppe Bildungsberichterstattung, 2016, S. 28; Weishaupt, 2017a), und insofern kann der deutlich höhere Migrationsanteil an den Inklusionsschulen - vor allem bei den großstädtischen Grundschulen - als ein Hinweis auf eine insgesamt sozial eher benachteiligte Schülerschaft an Inklusionsschulen in Hessen angesehen werden.

\section{Zur Gesamtsituation sonderpädagogischer Förderung in Hessen}

Die Auswirkungen des zunehmenden Anteils von Schüler*innen mit einem sonderpädagogischen Förderbedarf in allgemeinen Schulen auf die Situation sonderpädagogischer Förderung insgesamt soll abschließend betrachtet werden (vgl. Tab. 6). Die Inklusionsanteile unterscheiden sich deutlich bei den verschiedenen sonderpädagogischen Förderschwerpunkten. Die Schüler*innen im Förderschwerpunkt „Emotionale und soziale Entwicklung“ werden fast zur Hälfte an allgemeinen Schulen unterrichtet, die im Förderschwerpunkt „Lernen“ nur zu gut einem Drittel. Der Inklusionsanteil im Förderschwerpunkt „Körperliche und motorische Entwicklung“ entspricht in etwa dem Inklusionsanteil von 27,8 Prozent über alle Förderschwerpunkte hinweg. Unterdurchschnittlich ist der Inklusionsanteil im sonderpädagogischen Förderschwerpunkt „Sprache“, obwohl er - wie auch der Förderschwerpunkt „Lernen“ im Bundesdurchschnitt einen überdurchschnittlichen Inklusionsanteil aufweist (vgl. Tab. 1).

6 Grundsätzlich besuchen Flüchtlingskinder mit geringen Deutschkenntnissen in Hessen zunächst Intensivklassen zum Erwerb deutscher Sprachkenntnisse. 
Zur Situation sonderpädagogischer Förderung in Hessen im Schuljahr 2016/17 |

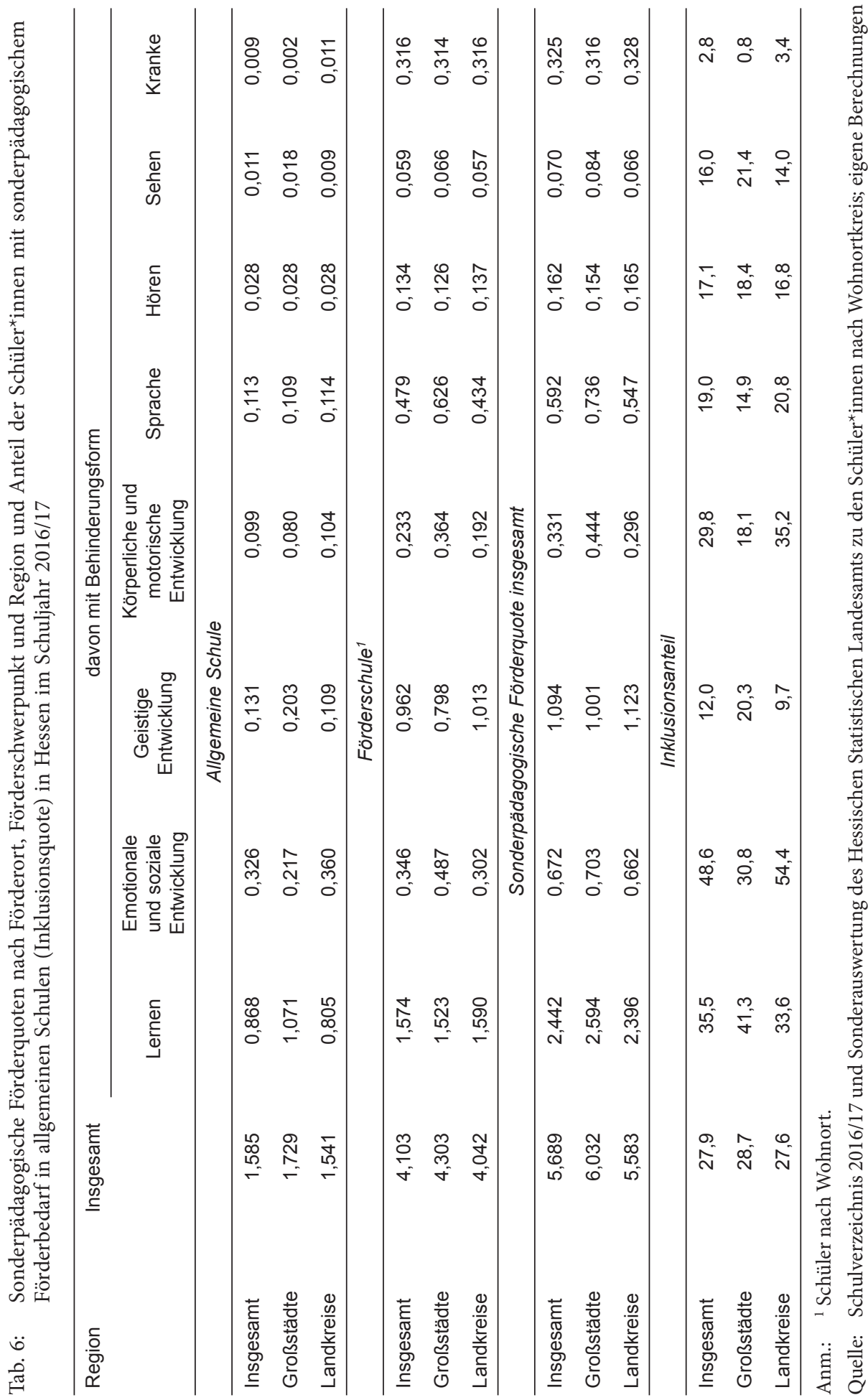


Im regionalen Vergleich gibt es bei den meisten sonderpädagogischen Förderschwerpunkten große Unterschiede in den Inklusionsanteilen. In den Landkreisen werden ein deutlich höherer Anteil - mehr als die Hälfte - der Schülerinnen im Förderschwerpunkt „Emotionale und soziale Entwicklung“ und ein erheblich unter den Großstädten liegender Anteil im Förderschwerpunkt „Lernen“ inklusiv unterrichtet. In den Großstädten wird von den Schüler*innen im Förderschwerpunkt „Geistige Entwicklung“ jede* $r$ fünfte an allgemeinen Schulen unterrichtet, in den Landkreisen nur jede ${ }^{\star} r$ zehnte. Durch einen hohen Inklusionsanteil in den Förderschwerpunkten „Emotionale und soziale Entwicklung“ und „Körperliche und motorische Entwicklung“ in den Landkreisen können die Unterschiede in den sonderpädagogischen Förderquoten zwischen Städten und Landkreisen etwas verringert werden.

Die in den Landkreisen niedrigeren Inklusionsquoten in den Förderschwerpunkten "Lernen“ und "Geistige Entwicklung" haben unterschiedliche Auswirkungen: Im Förderschwerpunkt „Lernen“ vergrößern sie die Stadt-Land-Unterschiede bei der sonderpädagogischen Förderquote, im Förderschwerpunkt „Geistige Entwicklung“ verringern sie sie. „Geistige Entwicklung“ ist der einzige Förderschwerpunkt, in dem die sonderpädagogische Förderquote der Landkreise deutlich über dem Landesdurchschnitt liegt.

Bei den sonderpädagogischen Förderschwerpunkten ohne ein in allen Kreisen bestehendes Förderschulangebot zeigte sich bereits, dass Unterschiede zwischen Schulstandortkreisen und Kreisen ohne Schulangebot in dem entsprechenden Förderschwerpunkt gewichtiger sind als Stadt-Land-Unterschiede. Inklusion bietet nicht zuletzt die Möglichkeit, in der Vergangenheit von einem Förderschulangebot nicht erreichte Schüler*innen außerhalb des Einzugsbereichs der Förderschulen sonderpädagogisch zu fördern (vgl. Tab. 7). Bisher wird diese Option aber nur in dem Förderschwerpunkt „Emotionale und soziale Entwicklung“ genutzt. In den anderen nicht flächendeckend versorgten Förderschwerpunkten sind auch die Inklusionsquoten in Kreisen ohne ein entsprechendes Förderschulangebot niedriger als in den Kreisen mit einem solchen Angebot. Damit wird das wichtige Ziel einer besseren wohnortnahen sonderpädagogischen Förderung in allen Förderschwerpunkten bisher in Hessen noch nicht erreicht. Eine mögliche Ursache könnte in den Regionen ohne eine entsprechende Förderschule in der fehlenden Verfügbarkeit von Förderschullehrkräften mit Lehrbefähigungen in den entsprechenden Förderschwerpunkten liegen. Dies wäre aber ein Anzeichen für eine konzeptionslose Entwicklung der Inklusion, denn sie kann ihre Ziele nur bei einer flächendeckenden Verfügbarkeit von Förderschullehrkräften in allen Förderschwerpunkten erreichen. 
Zur Situation sonderpädagogischer Förderung in Hessen im Schuljahr 2016/17 |

Tab. 7: Sonderpädagogische Förderquoten nach Städten/Kreisen mit oder ohne Förderschulstandort des jeweiligen Förderschwerpunkts in Hessen im Schuljahr 2016/17

\begin{tabular}{clcccccc}
\hline Schulart & Region & $\begin{array}{c}\text { Emotionale } \\
\text { und soziale } \\
\text { Entwicklung }\end{array}$ & $\begin{array}{c}\text { Körperliche } \\
\text { und } \\
\text { motorische } \\
\text { Entwicklung }\end{array}$ & Sprache & Hören & Sehen & Kranke \\
\hline $\begin{array}{c}\text { Allgemeine } \\
\text { Schule }\end{array}$ & Insgesamt & 0,336 & 0,102 & 0,116 & 0,029 & 0,012 & 0,009 \\
& Standortkreise & 0,310 & 0,102 & 0,119 & 0,028 & 0,018 & 0,014 \\
& Sonstige Kreise & 0,474 & 0,101 & 0,095 & 0,029 & 0,010 & 0,004 \\
\hline Förder- & Insgesamt & 0,375 & 0,240 & 0,494 & 0,139 & 0,061 & 0,362 \\
schule & Standortkreise & 0,403 & 0,346 & 0,555 & 0,228 & 0,100 & 0,458 \\
& Sonstige Kreise & 0,229 & 0,117 & 0,104 & 0,105 & 0,050 & 0,240 \\
\hline Insgesamt & Insgesamt & 0,711 & 0,341 & 0,610 & 0,167 & 0,073 & 0,371 \\
& Standortkreise & 0,713 & 0,448 & 0,674 & 0,256 & 0,118 & 0,472 \\
& Sonstige Kreise & 0,704 & 0,218 & 0,199 & 0,134 & 0,060 & 0,244 \\
\hline
\end{tabular}

Anm.: Berücksichtigt sind die Förderschüler*innen nach Wohnortkreis. Bei den Inklusionsschüle$\mathrm{r}^{\star}$ innen an allgemeinen Schulen wird davon ausgegangen, dass sie im Wohnortkreis die Schule besuchen. - Bei den Standortkreisen werden weniger als drei Schüler*innen in dem entsprechenden Schwerpunkt nicht als Schulstandort berücksichtigt.

Quelle: Schulverzeichnis 2016/17 und Sonderauswertung des Hessischen Statistischen Landesamts zu den Schüler*innen nach Wohnortkreis; eigene Berechnungen

\section{Schlussfolgerungen}

Die Analyse der schulischen sonderpädagogischen Förderung in Hessen anhand schulstatistischer Daten gibt einerseits Einblicke in unerwartete schulorganisatorische Konstellationen; andererseits wirft sie - da auf die Lehrkräftesituation an den Schulen nicht eingegangen werden konnte - vielfältige Fragen auf, die sich auf die Förderbedingungen an den Schulen und deren personelle Ausstattung beziehen. Bemerkenswert ist zunächst die Fördersituation für die Schüler*innen an einer großen Zahl von Förderschulen, die in ihrer Komplexität kaum in der wissenschaftlichen und öffentlichen Diskussion angesprochen wird. An vielen Förderschulen werden Schüler ${ }^{\star}$ innen mehrerer Förderschwerpunkte, z. T. zielgleich und z. T. zieldifferent, in kleinen nur einzügigen und jahrgangsgegliederten Schulen unterrichtet. Wie die Förderschulen diesen komplexen Förderbedingungen gerecht werden, ist bisher kaum erforscht. Die regionalen Disparitäten der schulischen Versorgung sind ein weiterer Problemaspekt des Förderschulsystems, in dem vor allem Regionen benachteiligt sind, die keine Standortkreise von Förderschulen des entsprechenden Förderschwerpunkts sind. Die Diagnose sonderpädagogischer Förderbedarfe scheint sich am regional verfügbaren Förderschulangebot zu orientieren. Andernfalls sind die 
zu beobachtenden erheblichen regionalen Unterschiede in den Förderquoten kaum erklärbar.

Für den nicht unerheblichen Teil der an Inklusionsschulen vereinzelten Schülerinnen mit einem sonderpädagogischen Förderbedarf erscheint es schwierig, adäquate sonderpädagogische Unterstützung des Lernprozesses zu erreichen. Es gibt aber auch einige Schulen, die mit einem Anteil von über fünf Prozent Inklusionsschülerinnen möglicherweise über den Schuleinzugsbereich hinaus Schülerinnen mit sonderpädagogischem Förderbedarf unterrichten. Damit würde das Ziel einer wohnortbezogenen sonderpädagogischen Förderung durch Inklusion eher durch das Konzept einer Schwerpunktschule ersetzt. Insgesamt ist keine zielorientierte Entwicklung der Inklusion erkennbar, die an den Eingangsklassen ansetzt und sich auf durch fehlende Förderschulen schlecht versorgte Regionen und die Förderschwerpunkte LES (Lernen, Emotionale und soziale Entwicklung, Sprache) konzentriert, wie dies naheliegend wäre.

Zwar haben Inklusionsschulen häufiger Ganztagsangebote als Schulen ohne Inklusionsschüler*innen; es sind an ihnen aber auch häufiger Intensivklassen für Flüchtlingskinder angesiedelt, und es sind Schulen mit einem überdurchschnittlichen Anteil von Schüler^innen mit Migrationshintergrund. Die Lehrkräfte an Inklusionsschulen sind dadurch mit vielfältigen pädagogischen Anforderungen konfrontiert, die die häufig geäußerte Kritik an der Umsetzung der Inklusion durchaus plausibel erscheinen lässt. Dazu trägt bei, dass den Schulen bisher kaum zusätzliche Ressourcen bei sozialen Benachteiligungen der Schüler*innen zur Verfügung gestellt werden und jüngste Studien sogar darauf hinweisen, dass diese Schulen personell schlechter versorgt sind als sozial nicht benachteiligte Schulen (Helbig \& Nicolai, 2019; Weishaupt, 2016).

Bei der vorliegenden Analyse konnten die allgemeinen Schulen in Hessen, die sowohl Förderschulzweige haben als auch Inklusionsschülerinnen unterrichten, nicht besonders berücksichtigt werden. Diese Schulen sind Beispiele für eine Vielfalt von schulorganisatorischen Konstellationen, die in den letzten Jahren entstanden sind und die über die Vorstellungskraft eines Außenstehenden hinausgehen. Deshalb wäre es wichtig, den konkreten Lern- und Förderbedingungen der Schüler*innen mit sonderpädagogischem Förderbedarf an den einzelnen Schulen mehr Aufmerksamkeit $\mathrm{zu}$ schenken und Standards zu entwickeln, die als Vorbedingungen sowohl an Förderschulen als auch an allgemeinen Schulen für eine angemessene sonderpädagogische Förderung erfüllt sein müssen. 


\section{Literatur und Internetquellen}

Autorengruppe Bildungsberichterstattung (2016). Bildung in Deutschland 2016. Ein indikatorengestützter Bericht mit einer Analyse zu Bildung und Migration. Bielefeld: Bertelsmann.

Autorengruppe Bildungsberichterstattung (2018). Bildung in Deutschland 2018. Ein indikatorengestützter Bericht mit einer Analyse zu Wirkungen und Erträgen von Bildung. Bielefeld: Bertelsmann.

Brüggemann, C., \& Tegge, D. (2016). Kennziffern in der Kritik: Anmerkungen zur indikatorengestützten Darstellung von Inklusion in der internationalen Bildungsberichterstattung. Zeitschrift für Inklusion. Zugriff am 17.03.2019. Verfügbar unter: https:// www.inklusion-online.net/index.php/inklusion-online/article/view/344/288.

Helbig, M., \& Nikolai, R. (2019). Bekommen die sozial benachteiligsten Schüler*innen die „besten“ Schulen? Eine explorative Studie über den Zusammenhang von Schulqualität und sozialer Zusammensetzung von Schulen am Beispiel von Berlin (Discussion Paper P-2019-002). Berlin: WZB. Zugriff am 18.03.2019. Verfügbar unter: https://bibliothek. wzb.eu/pdf/2019/p19-002.pdf.

Hessisches Statistisches Landesamt (2017). Die allgemeinbildenden Schulen in Hessen 2016, Teil 1: Grundschulen, Hauptschulen, Mittelstufenschulen, Förderstufen, Förderschulen, Sonderpädagogische Förderung an allgemeinen Schulen. Stand: 1. November 2016. Zugriff am 25.04.2018. Verfügbar unter: https://statistik.hessen.de/sites/statistik.hessen. de/files/BI1a_j16.pdf.

KMK (Sekretariat der Ständigen Konferenz der Kultusminister der Länder in der Bundesrepublik Deutschland) (2016a). Sonderpädagogische Förderung in allgemeinen Schulen (ohne Förderschulen) 2015/2016. Berlin \& Bonn: KMK.

KMK (Sekretariat der Ständigen Konferenz der Kultusminister der Länder in der Bundesrepublik Deutschland) (2016b). Sonderpädagogische Förderung in Förderschulen (Sonderschulen) 2015/2016. Berlin \& Bonn: KMK.

Moser, V., \& Lütje-Klose, B. (Hrsg.). (2016). Schulische Inklusion (Zeitschrift für Pädagogik, 62. Beiheft). Weinheim: Beltz.

Weishaupt, H. (2016). Schulen in schwieriger Lage und Schulfinanzierung. Die Deutsche Schule, 108 (4), 354-369. Zugriff am 18.03.2019. Verfügbar unter: https://www.waxmann. com/index.php?eID=download\&id_artikel=ART102057\&uid=frei.

Weishaupt, H. (2017a). Arme Kinder in der Grundschule: Die Situation in Rheinland-Pfalz. SchulVerwaltung. Ausgabe Hessen und Rheinland-Pfalz, 22 (5), 132-135.

Weishaupt, H. (2017b). Die Förderschule als sonderpädagogischer Förderort. In M. Gercke, S. Opalinski \& T. Thonagel (Hrsg.), Inklusive Bildung und gesellschaftliche Exklusion: Zusammenhänge - Widersprüche - Konsequenzen (S. 39-57). Wiesbaden: Springer VS. https://doi.org/10.1007/978-3-658-17084-4_4

Weishaupt, H. (2017c). Ganztagsgrundschulen in Hessen und Rheinland-Pfalz: Ein Blick auf die Zielverwirklichung. SchulVerwaltung. Ausgabe Hessen und Rheinland-Pfalz, 22 (10), 265-268.

Horst Weishaupt, Dr., geb. 1947, Prof. i. R.

E-Mail: weishaupt@dipf.de

Anschrift: DIPF | Leibniz-Institut für Bildungsforschung und Bildungsinformation, Rostocker Str. 6, 60323 Frankfurt am Main 